\title{
Predict Shame and Guilt Based on Self-differentiation and Defense Mechanisms
}

Iranian Evolutionary and Educational Psychology Journal June 2020: 98-109 (C) University of Hormozgan Publication 2020 DOI: 10.29252/ieepj.2.2.98 http://ieepj.hormozgan.ac.ir

\section{Maryam Rajabpour ${ }^{1}$, Banafsheh Omidvar $^{2 *}$}

\begin{abstract}
The purpose of this study was to predict shame and guilt based on self-differentiation and defense mechanisms. The research method is correlation. The statistical population of this study was students of Islamic Azad University of Pardis branch of Shiraz. Random cluster sampling method was used to select 140 people as the sample group. Participants responded to Self-Conscious Affect Inventory, Self-Differentiation (short form) Scale, and Self-Defense Styles inventory. Data were analyzed using multiple regression analysis with SPSS software. Findings showed that dimension of self-differentiation was a negative and significant predictor of feeling shame. In addition, the results showed that underdeveloped defense mechanisms were negative and significant predictors of guilt, and underdeveloped and psychosomatic defense mechanisms were positive and significant predictors of shame. Overall, it can be concluded that the effect of defense mechanisms and their differentiation dimensions on the sense of shame and guilt is important.
\end{abstract}

Keywords: defense mechanisms, guilt, self-conscious affect, self-differentiation, shame.

\section{Introduction}

Everyone encounters events in one's routine life which bring them a feeling of failure and incompetence. Following these experiences and self-evaluations, some emotions appear. Some of these emotions, such as shame and guilt are conscious that appear as a result of reflection and self-assessment (June Price Tangney, Stuewig, \& Mashek, 2007). Accordingly, since in the feelings of shame and guilt, the person self-assesses himself/herself, these two are categorized within the conscious emotions (J. Tangney \& Dearing, 2002; Tracy $\&$ Robins, 2004). In addition, as they play a role in ethical upbringing, they are identified as ethical emotions as well (J. Tangney \& Dearing, 2002). Moreover, the shame and guilt emotions are classified within the negative emotions as the people who experience them often feel unhappy, angry and irritable (Abbasi, Hosaini, $\&$ Golestaneh, 2017). Therefore, the two emotions of shame and guilt could be identified as conscious, social, ethical and negative feelings.

The conscious emotions are in fact the self-regulatory emotions that play a key role in directing the behavior,

1. MA Student, Islamic Azad university, Shiraz Branch, Shiraz, Iran

2. Assistant professor, Islamic Azad university, Shiraz branch, Shiraz, Iran

*Corresponding author email: omidvarpsy@gmail.com 
motivating the person to attend to the ethical and social standards and appropriate responses by providing feedback to the person about attitudes and behavior (Saeedi, Ghorbani, Sarafraz, \& Sharifian, 2012). The shame and guilt are irritating, self-evaluative and ethical emotions that are triggered by committing crimes, making mistakes, or failures in interpersonal issues (June Price Tangney, 1995).

Despite the public nature of these emotions, it is hard to conceptualize and define them. The reason for this complexity is that conscious emotions are affected by small and big cultural contexts and norms. For a long time, these two were not differentiated from each other. In fact, both of them are conscious feelings that are triggered by reflection and self-assessment that help self-regulation (Makogona \& Enikolopovb, 2013). These two have some commonalities but are quite different in terms of pathology and interpersonal relationships (Miceli \& Castelfranchi, 2018).

Some comparative studies cause disagreement in the inspiration consequences of shame and guilt (Makogona \& Enikolopovb, 2013). The emotion of shame is a negative experience that centers on the individual and forces him/her to do self-assessment. The emotion of shame incudes a sense of worthlessness, inability, and self-humiliation. For this reason, it is more painful than guilt. In fact, this emotion causes secrecy and encourages the person to escape from the embarrassing situation (June Price Tangney et al., 2007). In contrast, the guilt is a negative emotion that focuses on the behavior of the person. The individual blames oneself for doing something wrong (June Price Tangney et al., 2007). In the emotion of guilt, the focus is not on the person and negative self-evaluation is not done, but the focus is on the inappropriate behavior. Therefore, the emotion of guilt does not have a direct effect on the core identity of the person as he/she remains intact and healthy. The sense of guilt motivates the person to compensate and do things like apologizing, confessing, and positive social actions. Guilt is thus a constructive and ethical emotion as it leads to compensatory behavior. The emotion of shame has a close relationship with addiction, aggression, depression, suicide, and nutritional dysfunctions while the emotion of guilt has reverse relationships with these side effects.

Psychologist like Pierse and Singer (1953, as cited in June Price Tangney et al. (2007) have identified the emotion of guilt as a contrast between the ego and superego but the emotion of shame as the contrast between ego and the ideal ego which have roots in humiliation, fear of being abandoned and losing love. Lewis (1971) has deeply theorized the difference between the emotions of shame and guilt. He has emphasized that, in shame, the focus is on the self while in guilt the focus is on the wrong behavior. In recent years, this distinction has been conceptualized from cognitive views. For example, Tracy and Robins (2006) showed that the emotion of shame has positive correlations with inner, stable and uncontrollable documents about failure while the emotion of guilt had positive correlations with inner, unstable and controllable documents about failure.

The cognitive factors of shame and guilt are also consistent with the distinction of Miceli and Castelfranchi (2018) made between behavioral self-criticism and personal self-criticism. Theoretically speaking, the motions of shame are mostly related to the behavioral self-criticism. Shame is intertwined with the identity of the person and it is related with negative feelings about yourself. Therefore, shame is more painful than guilt and it includes the senses of inability and worthlessness (Makogona \& Enikolopovb, 2013). Very few studies have investigated the consequences of these two emotions. In Morris and Halford (2014), the results indicated that the insecure dependent children, especially the ones with dualist attachment style showed higher levels of shame than the ones with secure attachments.

The previous studies introduced shame as a completely incompatible emotion. Some other researchers have 
conducted theoretical and empirical studies and have reported that shame and guilt interact with each other in eastern culture where the norms are mostly communist and individuals are dependent on others and the function of shame is basically compatible (Gausel, Vignoles, \& Leach, 2016). Although some studies have been conducted to identify the predictor variables of shame and guilt, they have been limited. Self-discrimination and defensive mechanisms are among the variables related to mental health that seem to play a role in predicting the shame and guilt.

Self-discrimination is one of the fundamental concepts of Boen which states the personal independence accompanied by interpersonal intimacy in social relations. In other words, self-discrimination is a predictor variable in regulating the emotions, attitudes and feelings in interpersonal relationships, especially in stressful social situations (Shapiro, 2010). Self-discrimination means the potential for reflection when inner emotional pressures do not receive proper responses. Ability refers to flexibility and acting wisely even in stressful situations. Individuals who are not discriminated will get emotional more easily (Haefner, 2014). Their lives are influenced by reactions to other people. A discriminated person is able to find balance between rationale and emotions

This person is patient as well that is caused by resistance to emotional actions and reactions. Boen believes that social relationships consist of two powers capable of making balance in one's life: individuality and togetherness. Successful people's ability to make a balance between these two depend on their ability to control sensitivity or, as Boen puts it, their self-discrimination (Haefner, 2014). From the theoretical standpoint, at least four effective factors are involved in self-discrimination: emotional reactivity (a situation where the emotions dominate the rationale), emotional separation (declining or separating the problems when the person lives near the parents or when he lives far away or a mixture of both when the person envies close emotional relationship but escapes from it), integrating with other (Boen shows discrimination on a continuum where one end is discrimination and the other end is integration with others), and my location (having clear beliefs and norms in life).

Those who have low discrimination are more involved in defensive emotional mechanisms like emotional reactions, emotional rupture or integration with others. These people experience a more chronic stress in their relationships with others and reveal more physical and mental symptoms like physical and behavioral dysfunctions (Brown, 1999). It could be thus concluded that people with low self-discrimination experience more levels of shame as emotional rupture and chronic stress cause them to isolate more than others.

Ghasemzadeh-Barki and Khezri-Moghadam (2017) reported that women diagnosed with cancer who have more self-discrimination experience lower levels of shame and guilt and live a higher-quality life. In addition, Jamali Firoozabadi, Zarei, and Asadi (2011) stated that the linear combination of trust, shame and guilt could explain $58 \%$ of the variance in self-discrimination in the married students at Allame Tabatabei University in Tehran. Williamson, Sandage, and Lee (2007) found that hope plays a moderating role in the relationship between social relations and guilt while self-discrimination has a moderating role in social relations and shame. Moreover, defensive mechanisms could also play a role in managing these emotions. Shame, stress and fear are undesired emotions the effects of which are reduced by our brain using defensive mechanisms. Such mechanisms regulate the behaviors and are effective psychological tools that defend the people against threatening situations and correlate with the psychological well-being of the individuals (Cramer \& Jones, 2007). Such defenses change our emotions by changing our views or interpreting the reality but they do not 
change the reality (Frederickson, 2013). Many studies have reported in the past decades that suppressing the emotions and feelings could have negative physical, mental and social consequences. Declining the emotions not only reduces the potential to feel the negative reactions but also increase the possibility of destructive reactions against ourselves and others in a more complicated and sensitive way (Tracy \& Robins, 2004). Many classifications have been done for defensive mechanisms but the most well-known one has been introduced by Vaillant (1976). This researcher has classified them into Narcissistic (splitting, primary idealism, projection, replication, denial, and distortion), immature (outflowing, interrupting, introspecting, aggressive behavior, actionable, extrusion, and fantasy) photobiotic (controlling, moving, decomposing, extrusion, preventing, suppressing, reverse reaction, sexualizing, excusing, rationalizing, diminishing) and mature (sacrifice, altruism, prediction, austerity, comedy, idealism, and humility).

Defensive mechanism, especially the immature ones could hinder the cognition of reality and prevent the person from having a rational and effective defense and further reduce the cognitive and self-exploratory capacity of the individual. These mechanisms work in groups and are hardly employed individually. If they cannot bring a mental balance for the person, the social bond of the person will be lost and behavioral dysfunctions will appear. Zandi, Shahabinejan, and Borhan (2017) found that individual characteristics could function as sources of information in the analysis of psychological defenses. Ghamari, Rostami, Nader, and Ilbeigi (2010) reported that addicted people made more use of immature mechanisms while the non-addicted ones made use of mature ones.

Since shame and guilt are two effective variables in the adaptability and mental health of the individuals and few studies have been conducted on their meaning, growth, effective mechanisms and individual differences, this study made an effort to find if shame and guilt are predictable based on self-discrimination and defensive mechanisms.

\section{Material and Method}

The descriptive design of this study used regression analyses. The population included the students at Islamic Azad University in Shiraz studying in the second semester of the 2018-2019 educational year. Cluster random sampling was used to determine the sample of this study such that five faculties of humanities, economics and management, engineering 1 , engineering 2, and agriculture were randomly selected and two classes were randomly selected from each faculty. All the 140 selected participant from these classes responded to the instruments of this study. The researcher attended the classes as scheduled and the questionnaires were filled out after the students were informed of the purposes of the research and received instructions.

\section{Ethical considerations}

1. In this study, the respondents were reassured that their responses would be kept confidential.

2. The respondents were asked to answer the items of the questionnaire with honesty to obtain reliable results.

\section{Instruments}

The self-conscious emotion scale (TOSCA): This scale has been designed by June P Tangney, Wagner, Fletcher, and Gramzow (1992) which is a paper-and-pencil self-report scale that has 16 scenarios. The respondents read these situations about routine life and rank their behavioral and emotional responses to these situations. All the scenarios of this scale have items for shame and guilt while some items are about personal defensive behaviors like detachment and indifference. This questionnaire provides 16 life situations for the 
respondent which consist of 11 positive social situations and 5 negative ones. All the items are directly scored on a five-point Likert scale ranging from 1 (impossible) to 5 (quite possible). June P Tangney et al. (1992) approved the validity of this scale to assess the theoretical constructs of shame and guilt. Woien, Ernst, Patock-Peckham, and Nagoshi (2003) reported that this scale assesses the underlying concepts that the researcher intended to measure.

In Iran, the face validity of this scale has been approved by the experts in counselling and psychology. Roshan Chesli, Atrian Fard, and Noori Moghadam (2006) checked the concurrent validity of this scale by comparing it with GHQ-28 scale using 56 students. They found a correlation of .33 for this scale. In this study, the Cronbach's alpha estimate was found to be .72 for shame and .79 for the guilt scale.

The short form of discrimination scale (DSI-SH): This scale was designed by Drake (2011) and has 20 items for four sub-scales. These sub-scales include emotional reactivity, location of ego, emotional detachment and integration with others. The highest possible score is 120 and the lowest score is 20 . A high score indicates high self-discrimination and a low score shows low self-discrimination. Drake (2011) examined the reliability of these sub-scales using Cronbach's alpha and found the estimate of . 88 for the sub-scale of emotional reactivity, .85 for the location of ego, and .79 for integration with others.

In Iran, Fakhari, Latifian, and Etemd (2014) analyzed the psychometric features of this scale and found the Cronbach's alpha estimate of .78 for the whole scale, .67 for the location of ego, .48 for emotional reactivity, and .76 for integration with others. In addition, factor analyses confirmed the existence of four factors in the scale. In this study, Cronbach's alpha estimate for the whole scale was found to be .75 and the estimates for the sub-scales of ego, integration with others, emotional detachment and emotional reactivity were $.56, .59$, .58 , and .65 , respectively.

Defensive styles Questionnaire (DSQ-40): This scale was developed by Andrews, Singh, and Bond (1993). It was a revised version of the primary questionnaire earlier developed by Bond et al. in 1983. It has classified 20 defensive mechanisms in three developed, undeveloped, and mentally bothering factors. It is scored based on a nine-point Likert scale. Andrews et al. (1993) reported the test-retest reliability estimates between .46 and .86 and the Cronbach's alpha estimates of $.68, .58$, and .80 for the developed, mentally bothering, and undeveloped styles, respectively.

In Iran, Besharat (2012) reported the Cronbach's alpha estimates of .75, .73, and .74 for the developed, undeveloped and neurotic styles, respectively. They also found the test-retest reliability of .82 using a four-week time interval. In addition, Heidari Nasab (2006) reported the Cronbach's alpha of .71 for the whole scale using school students, .78 using university students and .74 for the whole population. The split-half reliability was found to be .53 as well. The results indicated appropriate validity and reliability for this questionnaire. The current study found Cronbach's alpha of .71 for the whole scale and estimates of .70, .46, and .44 for the developed, undeveloped and mentally bothering styles, respectively.

\section{Results}

The mean and standard deviation of each variable and its dimensions are reported in Table 1. The correlations between the variables are reported in Table 2 before answering the research questions to better understand the relationships between the variables. As indicated in Table 2, the defensive undeveloped mechanism and the mentally bothered defensive mechanism have positive significant relationships with shame. The location of ego, integration with others, and emotional reactivity have negative significant relationship with shame. In 
addition, the location of ego has a positive and significant relationship with guilt while the emotional reactivity has a negative and significant relationship with guilt. Moreover, guilt has a positive and significant relationship with shame.

Table 1. Mean and standard deviation of study variables

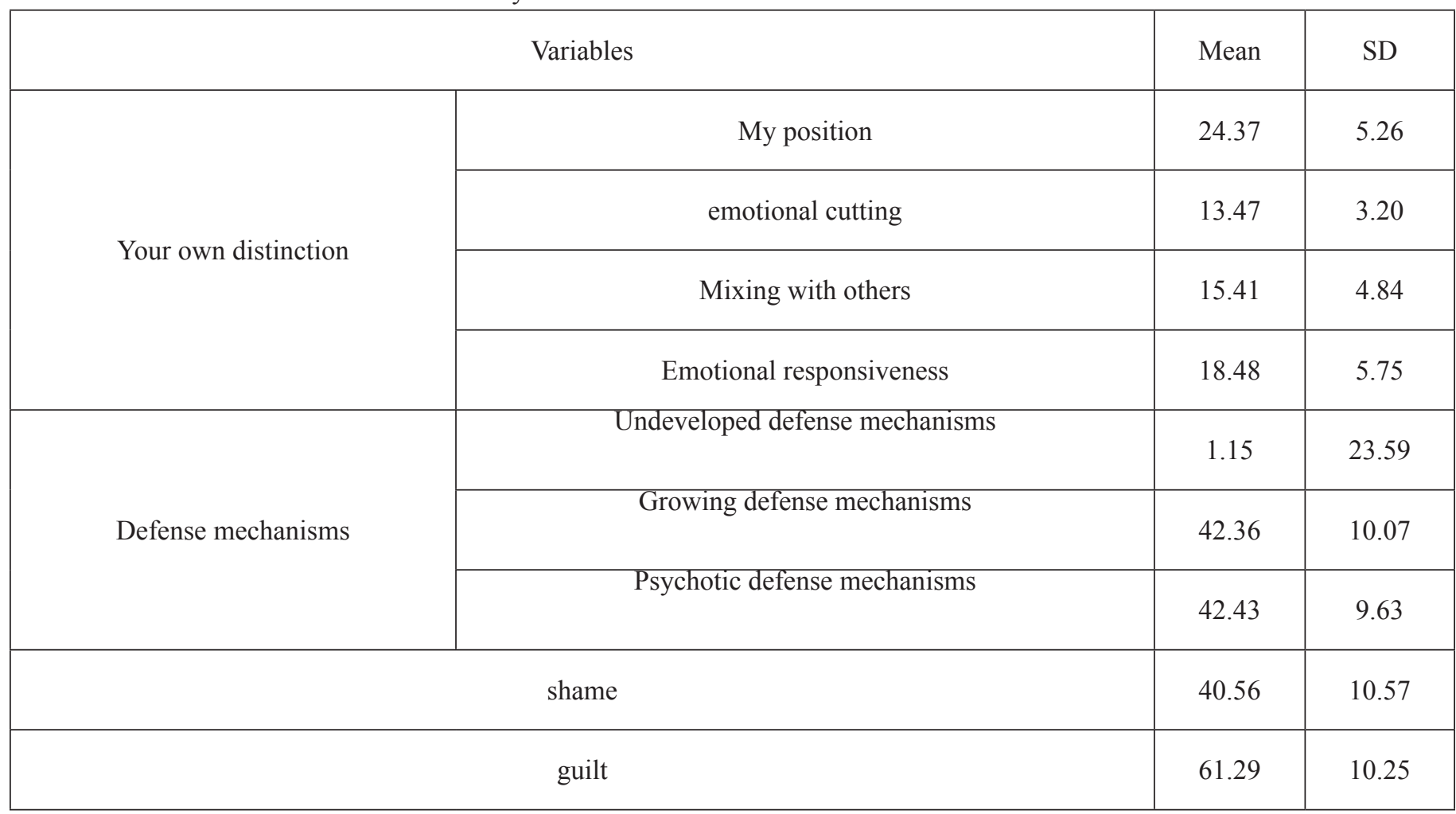

Table 2. Matrix of correlation between research variables

\begin{tabular}{|c|c|c|c|c|c|c|c|c|c|}
\hline \multicolumn{2}{|l|}{ Variables } & 1 & 2 & 3 & 4 & 5 & 6 & 7 & 8 \\
\hline \multirow{4}{*}{$\begin{array}{l}\text { Your own dis- } \\
\text { tinction }\end{array}$} & 1-My position & 1 & & & & & & & \\
\hline & 2-Mixing with others & $0.26^{* *}$ & 1 & & & & & & \\
\hline & 3-emotional cutting & -0.02 & 0.12 & 1 & & & & & \\
\hline & 4-Emotional responsiveness & $0.29 * *$ & $0.64 * *$ & 0.02 & 1 & & & & \\
\hline \multirow{3}{*}{$\begin{array}{l}\mathrm{D} \text { e } \mathrm{f} \text { e } \mathrm{n} \mathrm{s} \text { e } \\
\text { mechanisms }\end{array}$} & $\begin{array}{l}\text { 5- Undeveloped defense mecha- } \\
\text { nisms }\end{array}$ & $-0.27 * *$ & $-0.46 * *$ & -0.01 & $-0.45 * *$ & 1 & & & \\
\hline & 6-Growing defense mechanisms & $0.32 * *$ & 0.09 & 0.01 & 0.06 & 0.17 & 1 & & \\
\hline & 7-Psychotic defense mechanisms & -0.06 & $-0.40 * *$ & -0.06 & -0.15 & 0.17 & $0.23 *$ & 1 & \\
\hline \multicolumn{2}{|l|}{ 8- shame } & $-0.34 * *$ & $-0.36 * *$ & -0.06 & $-0.29 * *$ & $0.38 * *$ & 0.01 & $0.24 *$ & 1 \\
\hline \multicolumn{2}{|l|}{ Guilt } & $0.36^{* *}$ & -0.14 & -0.02 & $-0.18 *$ & -.10 & 0.17 & 0.16 & $0.24 * *$ \\
\hline
\end{tabular}


Stepwise multiple regression analysis was run to find the predictive power of self-discrimination and defensive mechanisms in emotions of shame and guilt. To do this, all the dimensions of self-discrimination and defensive mechanisms were inserted in the model of regression. The results are indicated in tables 3 and 4 .

Table 3. Analysis of shame prediction regression based on its differentiation dimensions and defense mechanisms

\begin{tabular}{|c|c|c|c|c|c|c|c|c|}
\hline Predictor variables & Steps & Independent variables & $\mathrm{R}$ & $\mathrm{R}^{2}$ & B & Beta & $\mathrm{t}$ & $\mathrm{p}$ \\
\hline \multirow{6}{*}{ Shame on you } & First & Mixing with others & 0.44 & 0.19 & -1.00 & -0.44 & 4.34 & 0.001 \\
\hline & \multirow{2}{*}{ Second } & Mixing with others & \multirow{2}{*}{0.49} & \multirow{2}{*}{0.24} & -0.87 & -0.38 & 3.78 & 0.001 \\
\hline & & My position & & & -0.48 & -0.23 & 2.30 & 0.024 \\
\hline & \multirow{3}{*}{ Third } & Mixing with others & \multirow{3}{*}{0.55} & \multirow{3}{*}{0.30} & -0.71 & -0.31 & 3.07 & 0.003 \\
\hline & & My position & & & -0.55 & -0.27 & 2.72 & 0.008 \\
\hline & & Psychotic defense mechanisms & & & 0.28 & 0.24 & 2.45 & 0.016 \\
\hline
\end{tabular}

The results of Table 3 showed that the predictor variables of shame entered the regression model in three steps. Accordingly, from among the dimensions of self-discrimination, only the two factors of integration with others and the location of ego were the significant negative predictors of shame and from among the dimensions of defensive mechanisms, only the mentally bothered style was the significant positive predictor of shame. The other dimensions were not identified as the predictor variables for the emotion of shame.

Table 4. Analysis of guilt prediction regression based on its differentiation dimensions and defense mechanisms

\begin{tabular}{|c|c|c|c|c|c|c|c|c|}
\hline Predictors & Steps & Independent variables & $\mathrm{R}$ & $\mathrm{R}^{2}$ & $\mathrm{~B}$ & Beta & $\mathrm{T}$ & $\mathrm{p}$ \\
\hline \multirow{6}{*}{ Feel guilty } & First & My position & 0.54 & .30 & 0.95 & 0.95 & 5.70 & 0.001 \\
\hline & \multirow{2}{*}{ Second } & My position & \multirow{2}{*}{0.61} & \multirow{2}{*}{0.37} & 1.13 & 0.65 & 6.64 & 0.001 \\
\hline & & Emotional responsiveness & & & -0.51 & -0.28 & 2.94 & 0.004 \\
\hline & \multirow{3}{*}{ Third } & My position & \multirow{3}{*}{0.63} & \multirow{3}{*}{0.40} & 1.09 & 0.62 & 6.44 & 0.001 \\
\hline & & Emotional responsiveness & & & -0.48 & -0.26 & 2.78 & 0.007 \\
\hline & & Psychotic defense mechanisms & & & 0.18 & 0.18 & 2.04 & 0.04 \\
\hline
\end{tabular}


in three steps. In the first step, the location of ego was the significant positive predictor for guilt. In the second step, the emotional reactivity was added to the model. This variable had a significant negative association with the emotion of guilt. The third variable which was entered in the third step was the mentally bothered defensive mechanism which significantly and positively predicted the feeling of guilt. Therefore, from among the predictor variables, the location of ego, emotional reactivity, and the mentally bothered defensive mechanism were respectively the strongest predictor variables for the feeling of guilt.

\section{Discussion}

As previously stated, the purpose of this study was to find the predictive role of shame and guilt based on self-discrimination and defensive mechanisms. The results indicated that the location of ego and integration with others were the significant negative predictors of shame. In addition, the location of ego was the significant positive predictor while the emotional reactivity was the significant negative predictor of guilt.

These findings are consistent with the results of Ghasemzadeh-Barki and Khezri-Moghadam (2017), Jamali Firoozabadi et al. (2011) and Bruno, Lutwak, and Agin (2009). Ghasemzadeh-Barki and Khezri-Moghadam (2017) stated that when the levels of self-discriminations increase, the levels of shame and guilt decrease resulting in a higher quality of life. Also, Jamali Firoozabadi et al. (2011) reported that people who had more self-discrimination had lower levels of shame.

To explain this result, we can state that those with higher levels of ego feel less ashamed as shame is associated with a negative attitude that distorts the identity of the person. Therefore, the more emotionally mature the person is, the less shame he/she will feel. Moreover, shame is accompanied with negative attitudes towards your characteristics and behaviors, so people with high levels of shame try to detach from others. This causes more psychiatric and interpersonal problems for the individual.

To explain the predictive power of self-discrimination in the feeling of guilt, we could say that self-discrimination increases generosity and mental health. Also, there is a close relationship between self-discrimination and stress, trust, close relations and emotion of guilt. The linear combination of trust, shame and guilt could explain the self-discrimination of married students which indicated the key role of this variable in the process of one's life (Jamali Firoozabadi et al., 2011). The positive relationship between ego and guilt could be explained with reference to the positive positon of guilt in our culture that could be viewed from a positive standpoint. In Iranian culture, the feeling of guilt is a way to put aside many ethical violations, while in the western culture, it is viewed as a mental problem. This feeling is part of our religious identity which is favored. A person who has a strong ego has clear beliefs in life and could thus avoid doing unethical actions. Additionally, people with high self-discrimination tend to compensate their wrong deeds and maintain their social relations accordingly. This characteristic is thus effective in the conscious emotion of guilt and motivates the person to compensate for the guilty behavior.

In the current study, it was revealed that the emotional reactivity was the significant negative predictor for the emotion of guilt. This is in line with Pearsaghi, Nazari, Hajihasani, and Nadalipour (2014). They stated that conscious control of stress is the base for self-discrimination, so the instruction of self-discrimination reduces the levels of stress in interpersonal relations and creates an opportunity for a strong ego and expression of personal beliefs. The negative relationship between emotional reactivity and guilt could be explained with the collectivist culture of Iranians. In this culture, every person seeks his identity in relation with others, so people 
need to control their emotions to be in social groups.

It was expected that the dimension of integration with others should have positively predicted the dependent variable but this was not the case. This could be explained with reference to the dynamic nature of self-discrimination such that people may appear self-discriminated in some situations and in relation with certain individuals but not in others. Therefore, the level of self-discrimination changes depending on the situation and audience.

This study also found that mentally bothered defensive mechanisms were the significant positive predictors for shame. This supports the results of Laurent et al. (2014) that defensive mechanisms protect self-confidence against shame, threat and narcissism by providing a sense of security in threatening situations and distance the person from external dangers. Few studies have been conducted for the prediction of shame made by defensive mechanisms. Ghamari et al. (2010) reported that people lose their ability to analyze, make decisions, and select appropriate behavior in difficult and stressful situations which, in turn, increase the possibility of incompatible behaviors. One example of immature or mentally bothered defensive mechanism in response to such problematic situations is the use of drugs.

One possible explanation for this is that people cannot make decisions in difficult and stressful situations and resort to immature and mentally bothered defensive mechanisms. Those who have more sense of shame use more mentally bothered defensive mechanisms. Such mechanisms either distort or convert the emotions as in opposite reactions where aggressive behavior turns into a more acceptable affection. For those who use mature defensive styles, pleasure reaches the maximum levels and conscious awareness of emotions and ideas will be the outcome, resulting in lower levels of shame. Shame is accompanied with humiliation and worthlessness, so the brain tries to use defensive mechanisms to reduce the negative feelings and manage the bothering emotions and relieve the undesired feelings. The management of more disturbed emotions will be more difficult, so the person may resort to immature defensive behaviors to reconstruct the emotional balance.

The results also showed that the mentally bothered defensive mechanisms were the positive and significant predictors of guilt, but since these mechanisms are negatively associated with the understanding of affections and their control, they distort or convert the cognition of emotions like the one occurs in opposite reaction where aggressive behavior turns into a more acceptable affection. Some of the defensive mechanisms change the center of attention (Pellitteri, 2002). Those who use the immature and mentally bothered defensive mechanisms could not manage their emotions well (Elias, Tobias, \& Friedlander, 2011).

Moreover, when experiencing the emotion of guilt, individuals use the mentally bothered defensive mechanisms and usually tend to compensate for the wrong deeds by apologizing and doing something in return. Overall, the results indicated that self-discrimination and defensive mechanisms could predict the conscious emotions of shame and guilt. It can be interpreted that these variables could assist the classification and discrimination of these conscious emotions.

This study had a number of limitations. First, the sample only consisted of university students, so the generalization of the results is limited. Second, the instruments of this study were only questionnaires which could have influenced the results. Moreover, some intervening variables like test anxiety and emotions were not controlled.

The results suggest that universities hold seminars, workshops and provide brochures to improve the 
developed defensive mechanisms and high self-discrimination to enhance the welfare of the students. The mental health experts are also recommended to use the results of this study in their clinical affairs to improve the mental health of their patients.

Further studies could investigate the moderating role of variables like education, economic and social status.

\section{Declaration of Conflicting Interests}

The author(s) declared no potential conflicts of interest with respect to the research, authorship, and/or publication of this article.

Funding: The author(s) received no financial support for the research, authorship, and/or publication of this article.

Acknowledgements: We would like to thank all the participants for their cooperation in filling out the questionnaires.

\section{References}

Abbasi, N., Hosaini, F., \& Golestaneh, M. (2017). The role of attributional styles in the conscious emotions of shame and guilt in elementary school students. Journal of Teaching and Learning Studies, 8(2), 1-18. doi:10.22099/jsli.2017.3967

Andrews, G., Singh, M., \& Bond, M. (1993). The Defense Style Questionnaire. Journal of Nervous and mental Disease.

Besharat, M. (2012). Reliability, Validity and Factor Analysis of Defensive Styles Questionnaire. Contemporary psychology(8).

Brown, J. (1999). Bowen family systems theory and practice: Illustration and critique. Australian and New Zealand Journal of Family Therapy, 20(2), 94-103.

Bruno, S., Lutwak, N., \& Agin, M. A. (2009). Conceptualizations of guilt and the corresponding relationships to emotional ambivalence, self-disclosure, loneliness and alienation. Personality and Individual Differences, 47(5), 487-491.

Cramer, P., \& Jones, C. J. (2007). Defense mechanisms predict differential lifespan change in self-control and self-acceptance. Journal of Research in Personality, 41(4), 841-855.

Drake, J. R. (2011). Differentiation of self inventory-short form: creation and initial evidence of construct validity. University of Missouri--Kansas City.

Elias, M. J., Tobias, S. E., \& Friedlander, B. S. (2011). Emotionally intelligent parenting: How to raise a self-disciplined, responsible, socially skilled child: Harmony.

Fakhari, N., Latifian, M., \& Etemd, J. (2014). A Study of Psychometric Properties of the Executive Skills Scale for Pre-School Children (Parent Form). Quarterly of Educational Measurement, 4(15), 35-58.

Frederickson, J. (2013). Co-creating change: Seven Leaves Press.

Gausel, N., Vignoles, V. L., \& Leach, C. W. (2016). Resolving the paradox of shame: Differentiating among specific appraisal-feeling combinations explains pro-social and self-defensive motivation. Motivation and Emotion, 40(1), 118-139.

Ghamari, H., Rostami, M., Nader, M., \& Ilbeigi, G., Reza. (2010). The Comparison of Personality Traits and Defense Mechanisms in Addicts Subjects and Healthy Subjects. Research on Addiction, 4(15), 71-82.

Ghasemzadeh-Barki, S., \& Khezri-Moghadam, N. (2017). The Role of Self-differentiation and Self-conscious 
of Shame and Guilt in Predicting the Quality of Life of Women with Breast Cancer. Iranian quarterly journal of breast disease, 10(1), 39-47.

Haefner, J. (2014). An application of Bowen family systems theory. Issues in Mental Health Nursing, 35(11), 835-841.

Heidari Nasab, L. (2006). Comparison of defense mechanisms in clinical and nonclinical samples according to standardization and findings based on psychomotricity of questionnaire of defense styles. A PhD thesis. Tarbiat Modarres University.

Jamali Firoozabadi, M., Zarei, S., \& Asadi, Z. (2011). Investigating the relationship between trust, shame and guilt with self-differentiation among married students. Psychological Research, 3(11), 15-26.

Laurent, A., Aubert, L., Chahraoui, K., Bioy, A., Mariage, A., Quenot, J.-P., \& Capellier, G. (2014). Error in intensive care: psychological repercussions and defense mechanisms among health professionals. Critical Care Medicine| Society of Critical Care Medicine, 42(11), 2370-2378.

Lewis, H., Block. (1971). Shame and guilt in neurosis. Psychoanalytic review, 58(3), 419-438.

Makogona, I. K., \& Enikolopovb, S. N. (2013). Problems with the assessment of shame and guilt. Psychology in Russia: State of the Art,(6), 4, 168-175.

Miceli, M., \& Castelfranchi, C. (2018). Reconsidering the differences between shame and guilt. Europe's journal of psychology, 14(3), 710.

Morris, M., \& Halford, W. K. (2014). Family mediation: A guide for family therapists. Australian and New Zealand Journal of Family Therapy, 35(4), 479-492.

Pearsaghi, F., Nazari, A., Hajihasani, M., \& Nadalipour, H. (2014). The effectiveness of self-differentiation therapy on the expression of female students at Allameh Tabatabai University in Tehran. Clinical Psychology, 16(4), 19-34.

Pellitteri, J. (2002). The relationship between emotional intelligence and ego defense mechanisms. The Journal of psychology, 136(2), 182-194.

Roshan Chesli, R., Atrian Fard, M., \& Noori Moghadam, S. (2006). Validation of Third Edition of the TOSCA to measure shame and guilt. Biomonthly Scientific Research of Shahed University, 31-46.

Saeedi, Z., Ghorbani, N., Sarafraz, M. R., \& Sharifian, M. H. (2012). The Relationship between Self-Compassion, Self-esteem and Self-Conscious Emotions Regulation. Journal of Research in Psychological Health, 6(3), 1-9.

Shapiro, M. (2010). An exploration of neurophysiological and cognitive processes associated with differentiation of self. (Doctor of Philosophy), The Pennsylvania State University, Pennsylvania

Tangney, J., \& Dearing, R. (2002). Shame and guilt. New York, NY. Guilford Press.

Tangney, JP, Stuewig, J., \& Mashek, DJ (2007). Moral emotions and moral behaviour. Annual Review of Psychology, 58, 345-372.

Tangney, J. P. (1995). Recent advances in the empirical study of shame and guilt. American Behavioral Scientist, 38(8), 1132-1145.

Tangney, J. P., Stuewig, J., \& Mashek, D. J. (2007). What's moral about the self-conscious emotions. The self-conscious emotions. Theory and research, 21-37.

Tangney, J. P., Wagner, P., Fletcher, C., \& Gramzow, R. (1992). Shamed into anger? The relation of shame and guilt to anger and self-reported aggression. Journal of personality and social psychology, 62(4), 669.

Tracy, J. L., \& Robins, R. W. (2004). “Putting the Self Into Self-Conscious Emotions: A Theoretical Model”. 
Psychological Inquiry, 15(2), 103-125.

Vaillant, G. E. (1976). Natural history of male psychological health: V. The relation of choice of ego mechanisms of defense to adult adjustment. Archives of General Psychiatry, 33(5), 535-545.

Williamson, I., Sandage, S. J., \& Lee, R. M. (2007). How social connectedness affects guilt and shame: Mediation by hope and differentiation of self. Personality and Individual Differences, 43(8), 2159-2170.

Woien, S. L., Ernst, H. A., Patock-Peckham, J. A., \& Nagoshi, C. T. (2003). Validation of the TOSCA to measure shame and guilt. Personality and Individual Differences, 35(2), 313-326.

Zandi, S., Shahabinejan, Z., \& Borhan, A. (2017). Predicting Defense Mechanisms based on Big Five Personality Traits among University Students. Zanko Journal of Medical Sciences, 18(56), 21-32. 